\title{
Medical Image of the Week: Septic Emboli from Elbow Abscess
}

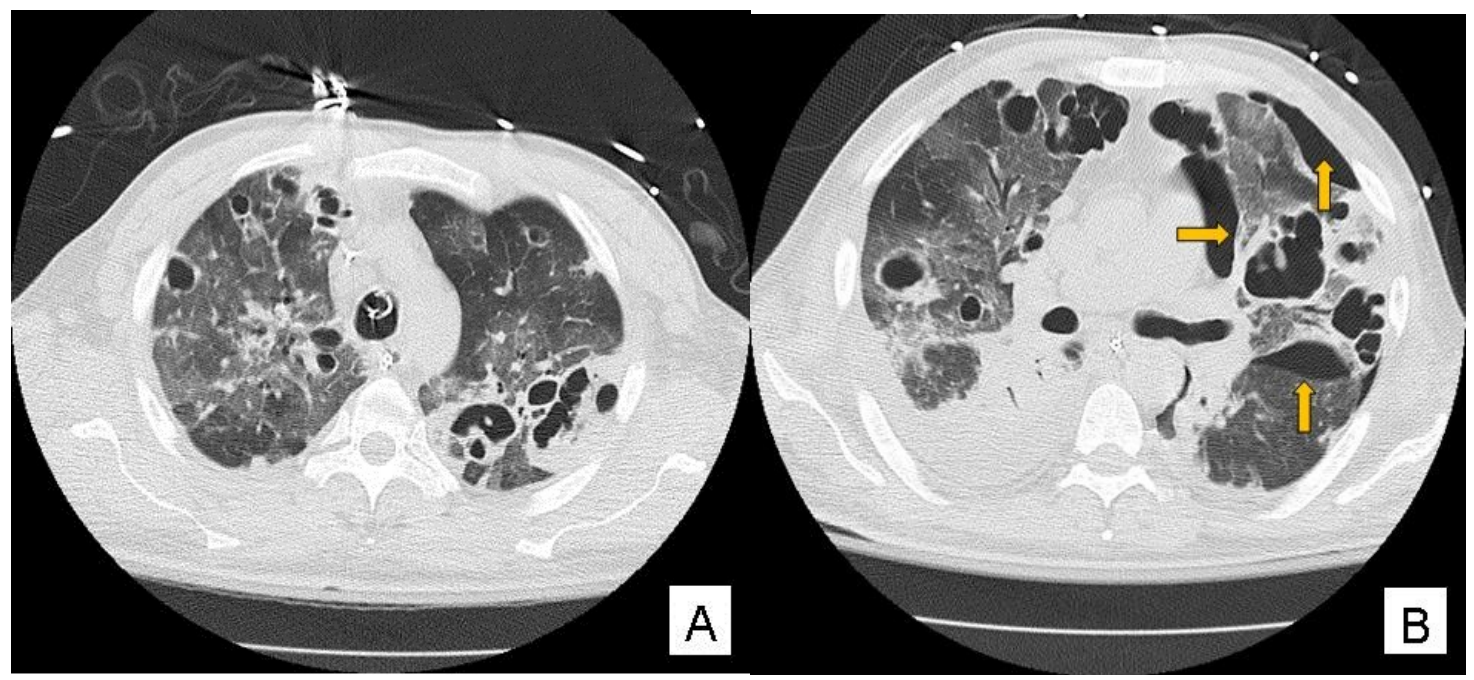

Figure 1. Panel A: Multiple thick wall cavities. Panel B: pneumothorax (arrows).

A 45 year old man with past medical history of rheumatoid arthritis and intravenous drug use presented with a several week history of progressive right elbow pain. He underwent incision and drainage with an operative diagnosis of septic arthritis. He developed postoperative respiratory failure requiring prolonged mechanical ventilation. Wound and blood cultures grew methacillinresistant Staphylococcus aureus. CT Chest revealed multiple thick walled cavities (A) from septic emboli as well as rupture of a pneumatocele causing a pneumothorax (B, arrows) necessitating chest tube insertion.

John F. Rosell, MD, Janet Campion, MD and Philip Factor, DO Departments of Medicine and Emergency Medicine University of Arizona

Tucson, AZ 